\title{
Mössbauer Spectroscopy and Transmission Electron Microscopy Analysis of Transition Carbides in Quenched and Partitioned Steel
}

\author{
D. T. Pierce ${ }^{1}$, D. R. Coughlin ${ }^{2}$, D. L. Williamson ${ }^{3}$, K. D. Clarke², A. J. Clarke ${ }^{2}$, J. G. Speer ${ }^{1}$, \\ D. K. Matlock ${ }^{1}$, E. De Moor ${ }^{1}$ \\ ${ }^{1}$ Advanced Steel Processing and Products Research Center, Colorado School of Mines, Golden, CO \\ ${ }^{2}$ Los Alamos National Laboratory, Materials Science and Technology Division, Los Alamos, NM \\ ${ }^{3}$ Department of Physics, Colorado School of Mines, Golden, CO
}

Quenching and partitioning (Q\&P) is a novel steel heat treatment that produces microstructures of martensite and retained austenite (Fig.1) [1]. Q\&P consists of quenching to a temperature (QT) between the martensite start and finish temperatures, partitioning at a temperature the same or greater than the QT, followed by quenching to room temperature (RT). The goal of the heat treatment is to partition carbon (C) from martensite to austenite, thereby stabilizing the austenite prior to the final quench. Competing reactions such as transition carbide formation can reduce the extent of $\mathrm{C}$ partitioning, resulting in less retained austenite and mechanical property variations. The small volume fractions, carbide thicknesses below $\sim 50 \mathrm{~nm}$, and numerous overlapping peaks makes X-ray diffraction characterization of transition carbides challenging. In contrast, Mössbauer spectroscopy (MS) with correlative transmission electron microscopy (TEM) is better suited for identifying and quantifying carbides. Most MS studies on transition carbides have focused on quenched and tempered microstructures in binary Fe-C steels with high $\mathrm{C}$, extensive amounts of carbides, and MS spectra primarily comprised of resonance from a limited number of unique Fe sites [2]. Q\&P steels with lower C and carbide fractions, alloying additions of Manganese $(\mathrm{Mn})$, Silicon ( $\mathrm{Si}$ ), and other elements, and significant amounts of retained austenite in the microstructures have more complex MS spectra, requiring more precise analysis methods.

A $0.38 \mathrm{C}-1.54 \mathrm{Mn}-1.48 \mathrm{Si}$ wt.\% steel underwent austenitization, quenching to $225{ }^{\circ} \mathrm{C}$ and partitioning at $400{ }^{\circ} \mathrm{C}$ for 10 or $300 \mathrm{~s}$. Microstructural characterization using a Philips CM200 TEM operated at $200 \mathrm{kV}$ indicates the presence of $\eta$-carbides (Fig.2). The (110) $\eta$ super lattice reflections in the selected area diffraction pattern in Fig.2b arise from the ordering of $\mathrm{C}$ atoms in the octahedral sites of the orthorhombic $\eta$-carbide structure [2,3]. The MS spectra in Fig.3 were acquired at RT with a ${ }^{57} \mathrm{Co}-\mathrm{Rh}$ source and a spectrometer operating in the triangular constant acceleration mode. Resonance due to martensite, austenite and $\eta$-carbide are present in the MS spectra. Five sextets with varying hyperfine fields and other sextet fitting parameters accounted for the total resonance of the complex distribution of Fe sites in the martensite [3]. Resonance due to Fe sites in austenite with zero $\mathrm{C}$ first or second nearest neighbors $(\mathrm{A}(0,0))$ and with zero $\mathrm{C}$ first but with $n(n=1,2,3$ or 4$) \mathrm{C}$ second nearest neighbors $(\mathrm{A}(0, \mathrm{n}))$ were fit with two singlets [4]. Resonance due to Fe sites in austenite with one or two C first nearest neighbors (A(c)) was fit with a quadrupole split doublet [4]. The MS spectra also reveal magnetic six line resonances from two different Fe sites in $\eta$-carbide: 1) Fe sites with three $\mathrm{C}$ nearest neighbors corresponding to stoichiometric $\eta$-carbide, $C\left(\eta_{s}\right)$, and 2) Fe sites with two nearest $C$ neighbors corresponding to non-stoichiometric $\eta$-carbide, $C\left(\eta_{\mathrm{ns}}\right)$, [3]. Fig.4 shows the MS spectrum of the $300 \mathrm{~s}$ sample on an expanded scale. The influence of both $\eta$-carbide components on the spectrum is evident near the resolved 2 and 5 lines (labeled in Fig.3) of each resonance in Fig.4. $\eta$-carbide fractions increase with increasing partitioning time. A correction [3] accounted for the saturation of the absorption spectrum due to sample thicknesses of $\sim 30$ $\mu \mathrm{m}$, which disproportionately attenuates the martensite and austenite resonance relative to $\eta$-carbide. We calculated the recoilless fraction for $\eta$-carbide, a fundamental parameter in quantitative MS analysis, to be 0.88 . The recoilless fraction of $\eta$-carbide is significantly greater than that for martensite and austenite ( $\sim 0.81)$, making use of this parameter important for quantitative MS investigations [3]. The total fractions of $\eta$-carbide, including $\eta_{\mathrm{s}}$ and $\eta_{\mathrm{ns}}$ components, were $1.4 \pm 0.3$ and $2.4 \pm 0.3$ at. $\%$ after partitioning for 10 and $300 \mathrm{~s}$, respectively. Therefore, $\eta$-carbides consume a significant portion ( 24 and $41 \%)$ of the bulk C [5]. 


\section{References:}

[1] J. G. Speer, D. V. Edmonds, F. C. Rizzo, D. K. Matlock, Curr Opin Solid St M 8 (2004), p. 219.

[2] D. L. Williamson, K. Nakazawa, G. Krauss, Metall Trans A 10 (1979), p. 1351.

[3] D. T. Pierce, D. R. Coughlin, et al., Acta Mater., http://dx.doi.org/10.1016/j.actamat.2015.01.024

[4] K. F. Laneri, J. Desimoni, G. J. Zarragoicoechea, et al., Phys Rev B 66;134201 (2002), p. 1.

[5] This work was supported by the U.S. Department of Energy under Award Number DE-EE0005765. The support of the ASPPRC sponsors is gratefully acknowledged. DRC, KDC, and AJC acknowledge support from Los Alamos National Laboratory, operated by Los Alamos National Security, LLC, under Contract DE-AC52-06NA25396 for the U.S. Department of Energy.

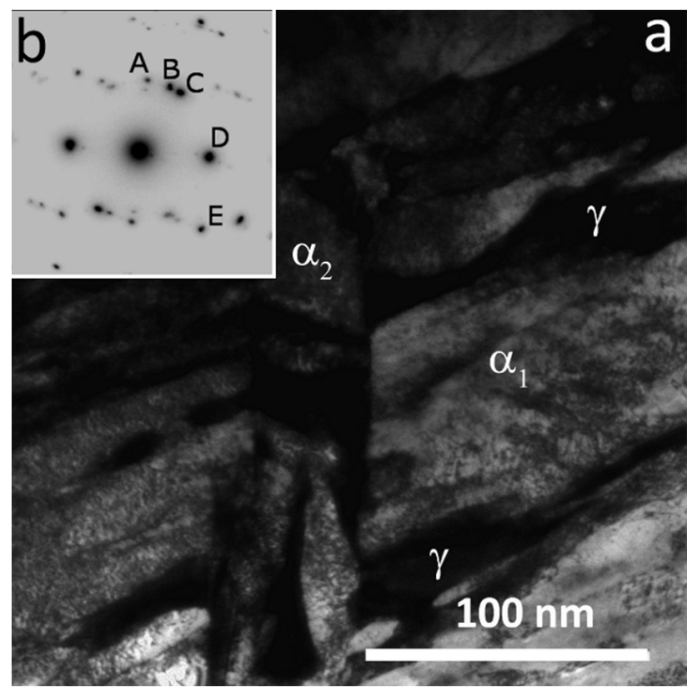

Fig.1 (a) Bright-field (BF) TEM image of two martensite variants with zone axes of $[-111]_{\alpha 1}$ and $[001]_{\alpha_{2}}$ and an austenite film with a zone axis of $[011]_{\gamma}$ after partitioning at $400^{\circ} \mathrm{C}$ for $300 \mathrm{~s}$. (b) Inset SAD pattern: $\mathrm{A}=(-110)_{\alpha 2}, \mathrm{~B}=(-11-1)_{\gamma}, \mathrm{C}=(110)_{\alpha 1}$, $\mathrm{D}=(11-1)_{\gamma},(101)_{\alpha 1,},(110)_{\alpha 2}$, and $\mathrm{E}=(200)_{\alpha 2}$.

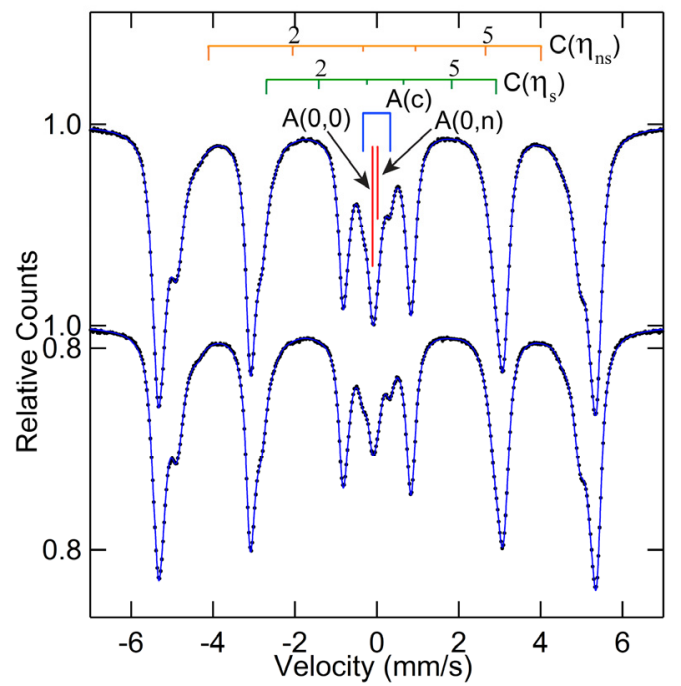

Fig.3 MS spectra: relative counts as a function of source velocity for samples partitioned at $400{ }^{\circ} \mathrm{C}$ for 10 (top) and $300 \mathrm{~s}$ (bottom). The stick diagrams represent the $A(0,0), A(0, n), A(C), C\left(\eta_{s}\right)$ and $C\left(\eta_{n s}\right)$ resonances and are not to scale. Martensite components have been removed for clarity.

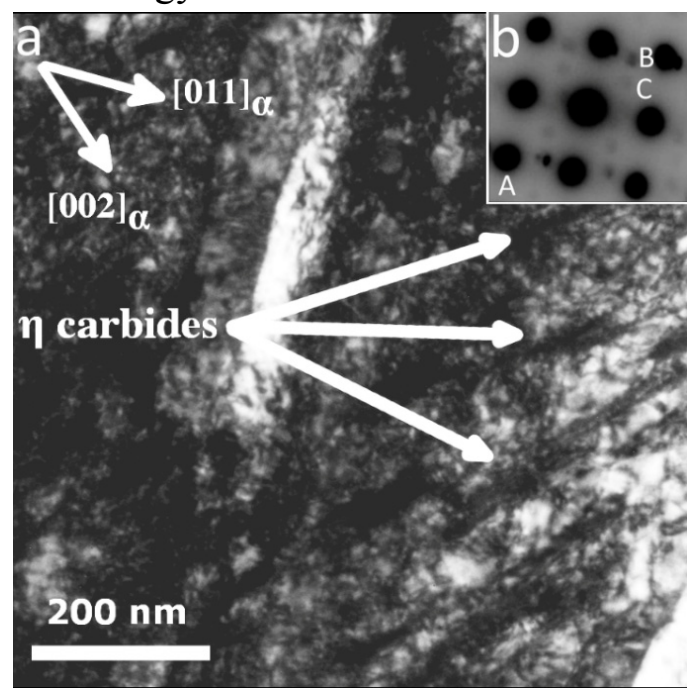

Fig.2 (a) $[-100]_{\alpha}$ zone axis BF TEM micrograph showing $\eta$-carbides within the martensite matrix in a sample partitioned for $10 \mathrm{~s}$ at $400{ }^{\circ} \mathrm{C}$, and (b) $[-100]_{\alpha}$ SAD pattern corresponding to the image in (a) where $\mathrm{A}=(0-20)_{\alpha}, \mathrm{B}=(210)_{\eta}$, and $\mathrm{C}=(110)_{\eta}$ indicate superlattice reflections.

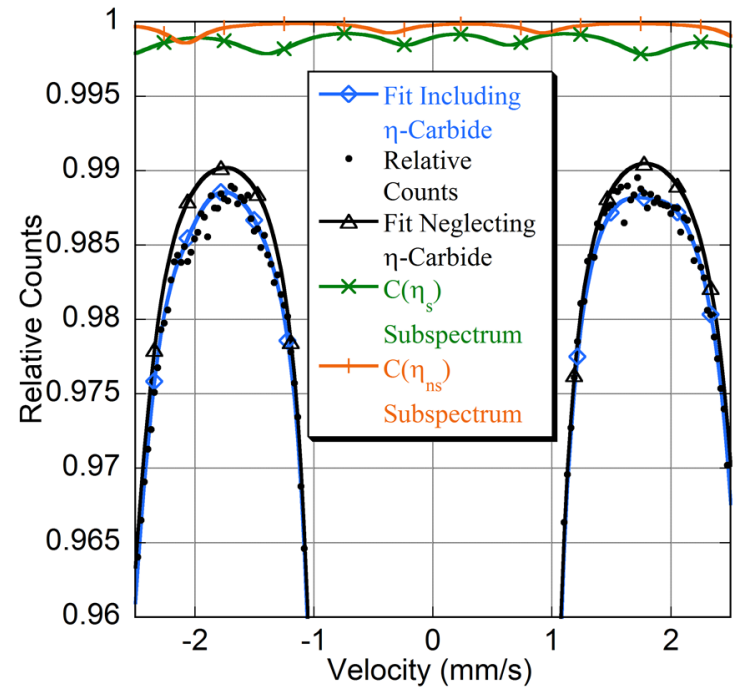

Fig.4 Expanded scale MS spectrum for the sample partitioned for $300 \mathrm{~s}$ from Fig.3. Relative counts, $\eta_{\mathrm{s}}$-and $\eta_{\mathrm{ns}}$-carbide subspectra, and fit including and neglecting $\eta$-carbide for the sample partitioned at $400{ }^{\circ} \mathrm{C}$ for $300 \mathrm{~s}$. The quality of fit improves with the inclusion of the $\eta_{\mathrm{s}}$-and $\eta_{\mathrm{ns}}$-carbide subspectra. 